\title{
Computational analysis reveals the coupling between bistability and the sign of a feedback loop in a TGF- $\beta 1$ activation model
}

Huipeng Li $i^{1,2}$, Lakshmi Venkatraman ${ }^{1,2}$, Balakrishnan Chakrapani Narmada ${ }^{2,3,7}$, Jacob K. White ${ }^{1,5}$, Hanry Yu ${ }^{1,2,3,4,6,7,8^{*}}$ and Lisa Tucker-Kellogg ${ }^{1,9,10^{*}}$

From 16th International Conference on Bioinformatics (InCoB 2017)

Shenzhen, China. 20-22 September 2017

\begin{abstract}
Background: Bistable behaviors are prevalent in cell signaling and can be modeled by ordinary differential equations (ODEs) with kinetic parameters. A bistable switch has recently been found to regulate the activation of transforming growth factor- $\beta 1$ (TGF- $\beta 1$ ) in the context of liver fibrosis, and an ordinary differential equation (ODE) model was published showing that the net activation of TGF- $\beta 1$ depends on the balance between two antagonistic sub-pathways.

Results: Through modeling the effects of perturbations that affect both sub-pathways, we revealed that bistability is coupled with the signs of feedback loops in the model. We extended the model to include calcium and Krüppellike factor 2 (KLF2), both regulators of Thrombospondin-1 (TSP1) and Plasmin (PLS). Increased levels of extracellular calcium, which alters the TSP1-PLS balance, would cause high levels of TGF- $\beta 1$, resembling a fibrotic state. KLF2, which suppresses production of TSP1 and plasminogen activator inhibitor-1 (PAI1), would eradicate bistability and preclude the fibrotic steady-state. Finally, the loop PLS - TGF- $\beta 1$ - PAl1 had previously been reported as negative feedback, but the model suggested a stronger indirect effect of PLS down-regulating PAl1 to produce positive (double-negative) feedback in a fibrotic state. Further simulations showed that activation of KLF2 was able to restore negative feedback in the PLS - TGF- $\beta 1$ - PAl1 loop.

Conclusions: Using the TGF- $\beta 1$ activation model as a case study, we showed that external factors such as calcium or KLF2 can induce or eradicate bistability, accompanied by a switch in the sign of a feedback loop (PLS - TGF- $\beta 1$ - PAI1) in the model. The coupling between bistability and positive/negative feedback suggests an alternative way of characterizing a dynamical system and its biological implications.
\end{abstract}

Keywords: Bistability, Positive feedback, Computational modelling, ODEs, Dynamical systems, Biochemical network, TGF- $\beta 1$, Bifurcation analysis

\footnotetext{
* Correspondence: hanry_yu@nuhs.edu.sg; Lisa.Tucker-Kellogg@dukenus.edu.sg

${ }^{1}$ Computational and Systems Biology Program, Singapore-MIT Alliance,

Singapore 117576, Singapore

Full list of author information is available at the end of the article
} 


\section{Background}

Bistability has been found in many biological systems, and mathematical models using ordinary differential equations (ODEs) with respect to time provide a good representation for studying these dynamic behaviors [1-4]. Some bistable systems show a binary behavior at the level of single cells but exhibit a graded response for a population of cells [1], while other bistable systems cause a binary output even for populations of cells [5]. Different strategies have been developed to study the two different categories of bistable systems. For systems with bistability at the level of single cells, the bistable nature of the output is often wellknown, guiding researchers to elucidate the underlying molecular circuit that enables the bistability. For systems with population-level bistability, the bistability may not be obvious by inspection, in which case, laborious experimental measurement is required to establish the phenomenon [5]. In a recent study, we modeled the bistable activation of transforming growth factor- $\beta 1$ (TGF$\beta 1$ ), which belongs to the category of population-level bistability. The bistability was validated experimentally by showing hysteresis in an in vitro system [5]. TGF- $\beta 1$ is a cytokine with broad importance for contexts such as cancer, liver cirrhosis, wound healing and regeneration. Our model captures multiple pathways with positive and negative effects towards TGF- $\beta 1$ and its activators (Fig. 1; Additional file 1: Supplementary Note 1).

In the current study, we explore some possible triggers that could influence the bistable transition, and look for system properties that correlate with bistability. We demonstrate that factors like calcium and Krüppel-like factor 2 (KLF2) (Additional file 1: Supplementary Note 2) level can be modeled implicitly into the reaction rate parameters of the model, allowing us to discuss the on and off transitions of bistability under different

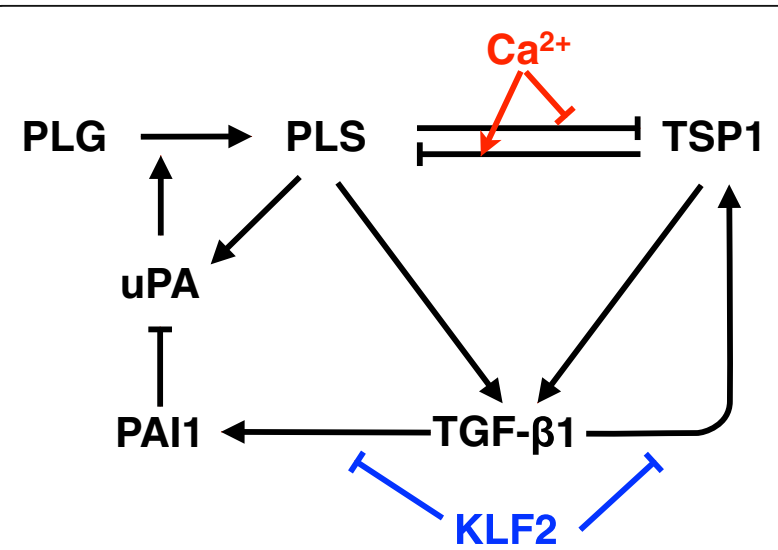

Fig. 1 TGF- $\beta 1$ bistable activation model. Black arrows represent the reactions from [5]. Red arrows represent the effects of calcium on the PLS-TSP1 interaction. Blue arrows represent the effects of KLF2 on PAI1 and TSP1 production. UPA is urokinase plasminogen activator, and PLG is plasminogen conditions. We identified a coupling between the sign of a signaling loop in the model (i.e., whether the signaling loop shows positive feedback or negative feedback) and the presence of bistability in the model. This could suggest alternative ways to identify and validate of systems with population-level bistability.

\section{Results}

Calcium and KLF2 have potential influence on the steady state of TGF- $\beta 1$ activation

Calcium would promote the steady state with high TGF- $\beta 1$ activation

We built the low and high calcium variants of the model by considering the potential effects of calcium on the PLS-TSP1 interaction (see Methods, Fig. 1 red arrows). Three parameters for the calcium effect were not known quantitatively and were estimated (see Methods). In Fig. 2a-b, we simulated the low calcium model and the high calcium model over time with 27 total initial configurations. These 27 configurations were combinations of 3 initial concentrations for each of TGF- $\beta 1$, TSP1, and plasmin, the ssT level, ssP level, and the mean level of ssT and $\operatorname{ssP}\left(27=3^{3}\right)$. The initial concentrations of other species were set to the average of their two steady state levels, (i.e., $0.5 \mathrm{ssT}+0.5 \mathrm{ssP}$ ). In the low-calcium model, all trajectories converged to ssP with low TGF$\beta 1$, but in the high-calcium model, several of the initial configurations converged to ssT with high TGF- $\beta 1$. To generalize our understanding of this effect, we plotted the boundary (the separatrix, Fig. 2c) between the initial configurations that caused convergence toward ssT (red) and the initial conditions that caused convergence toward ssP (blue). Initial concentrations were constants for all species other than PLS and TSP1. By comparing the separatrix of the low calcium model (dot) and the high calcium model (circle), we observe a shift of the separatrix toward the blue (ssP) region. This means the red (ssT) region is enlarged in high calcium environment (arrow 3). As expected, calcium tips the balance between PLS and TSP1 to achieve a significant effect on steady state of TGF- $\beta 1$ activation.

\section{KLF2 would eliminate the steady state with high TGF- $\beta 1$ activation}

KLF2 is a transcription factor studied extensively in atherosclerosis and fibrosis, and previous studies of KLF2 signaling showed TSP1 and PAI1 (plasminogen activator inhibitor-1) to be two of its most strongly affected targets $[6,7]$. To study how KLF2 would affect bistable activation of TGF- $\beta 1$, variants of the TGF- $\beta 1$ activation model were built as described in methods (Fig. 1 blue arrows). We built a model called "100\% KLF2" that downregulated the TSP1 production and PAI1 production rates, proportional to the published effects of KLF2 on 

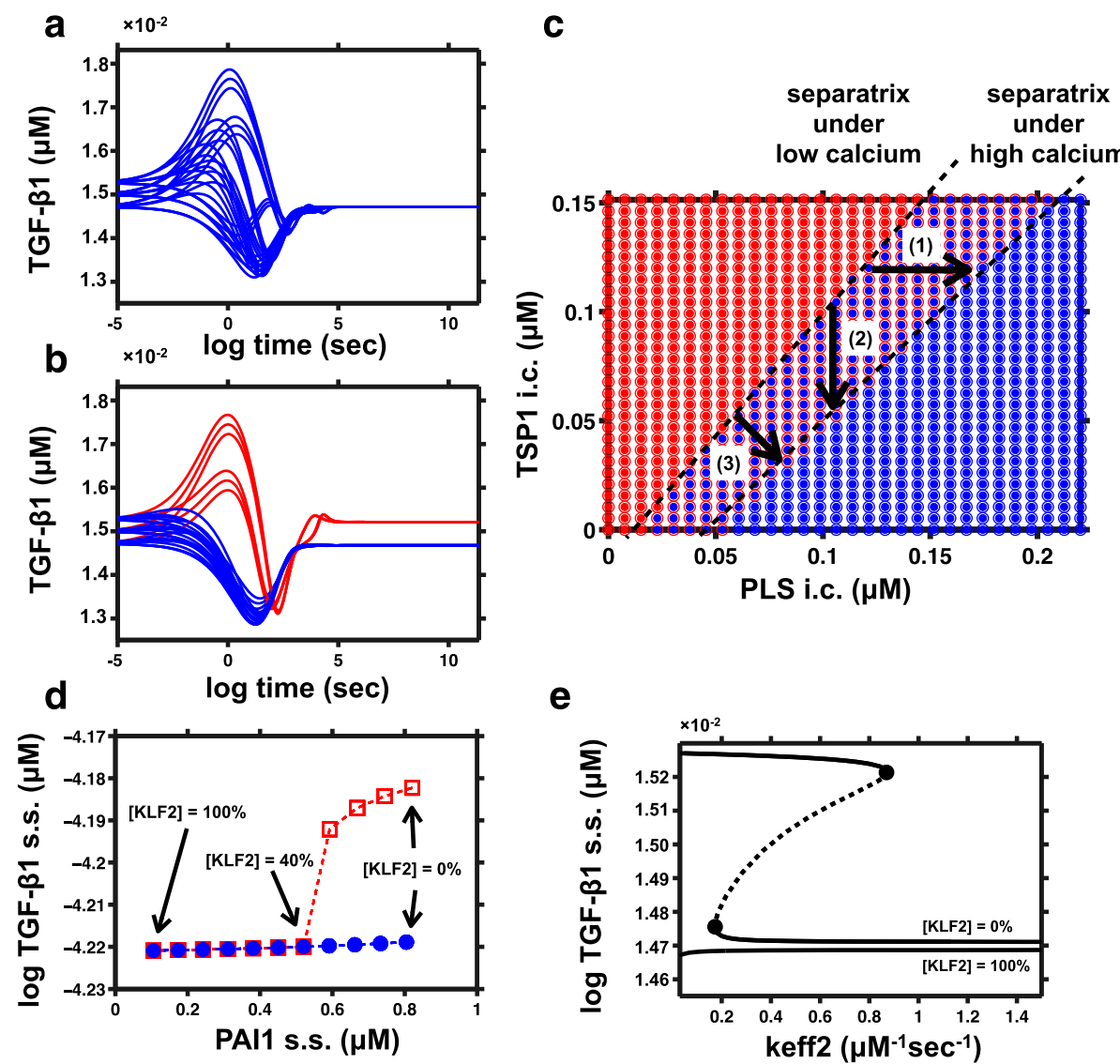

Fig. 2 Calcium and KLF2 have potential influence on the steady state of TGF- $\beta 1$ activation. $\mathbf{a}-\mathbf{b}$ TGF- $\beta 1$ abundance is plotted over time, after initializing the system from a given set of initial concentrations with (a) low calcium or (b) high calcium. In both cases, curves have been colored blue if they converge to a steady state with low TGF- $\beta 1$ activation (ssP), and colored red if they converge to a steady state with high TGF- $\beta 1$ activation (sST). c Calcium causes a shift in the separatrix between steady states. Low-calcium and high-calcium simulations were performed using various initial concentrations of PLS and TSP1. After observing in Fig. 2a-b that initial conditions with $0.5 \mathrm{ss} P$ and 0.5ssT were usually in the basin of convergence for the SSP state (for the low calcium model), we decided to shift the initial conditions. For studying the behavior of the separatrix, the initial concentrations were set to $.25{ }^{*} \mathrm{ssP}$ and $.75^{*} \mathrm{ss} T$. The steady state outcomes are shown by colors, with red indicating the steady state with high TGF- $\beta 1$ (sST), and blue indicating low TGF- $\beta 1$ (ssP). For each combination of PLS and TSP1, the low calcium result is indicated by the color of the small inner dot, and the high-calcium result is indicated by the color of the outer circle. $\mathbf{d}$ Two steady state (s.s.) levels of TGF- $\beta 1$ and PAl1 under different levels of KLF2. Red squares represent steady states with high TGF- $\beta 1$ activation (ssT), while blue dots represent steady states with low TGF- $\beta 1$ activation (ssP). The $y$-axis is the log of the steady state concentration of TGF- $\beta 1$. e Bifurcation analysis under KLF2 low and KLF2 high conditions

the mRNA levels of TSP1 (-7.8 fold) and PAI1 (-7.4 fold). This is a strong effect, so we also built models with $90 \%, 80 \%, \ldots 10 \%$, and $0 \%$ of the KLF2 effects on the TSP1 and the PAI1 production rates. Each model in the series was simulated to obtain the steady state concentrations. When a dynamical system is bistable, its two steady states are commonly obtained by simulating the model twice, once starting from each side of the separatrix boundary (for example, initializing the system with opposite extreme levels of TGF- $\beta 1$ ). For our series of models, the steady states obtained after initialization with high TGF- $\beta 1$ (resembling ssT) were plotted with open red boxes, and the steady states obtained after initialization with low TGF- $\beta 1$ (resembling ssP) were plotted with solid blue circles (Fig. 2d). Models for each level of KLF2 were plotted in terms of PAI1 and TGF- $\beta 1$ steady states (with KLF2 levels decreasing from left to right). For models with KLF2 $\geq 40 \%$, the open red boxes fell at the same points as the solid blue circles, indicating they are monostable. For models with KLF2 $\leq 30 \%$, the red open boxes were distinct from the blue circles, indicating two steady states. For KLF2 levels from $0 \%$ to $100 \%$ (right to left), the low TGF- $\beta 1$ steady state (blue dots) remained almost constant, while the high TGF- $\beta 1$ steady state (red boxes) merged with the low TGF- $\beta 1$ steady state in an ultrasensitive manner when the KLF2 effect increased from $30 \%$ to $40 \%$.

Bifurcation analysis studies how parameter change affects the qualitative behavior and the steady states of a system [8]. Bifurcation plot allows us to see all the 
equilibria of the system and how the equilibria vary with change of KLF2 levels and other rate parameters. We chose one parameter named "keff2" to show the steady state behavior of the low KLF2 and high KLF2 system. "Keff2", the enzymatic efficiency of plasmin, is one of many rate parameters that affect the overall bistability of the system. Figure 2e shows the bifurcation plot for the 0\% KLF2 model and the 100\% KLF2 model with respect to the parameter "keff2". Solids lines represent the stable steady states of the system (ssP or ssT). Dotted lines represent one unstable steady state between the two stable steady states, which is not achievable through simulation. Black circles represent two limit points in the bifurcation curve, which separates the monostable regime and bistable regime. Bifurcation analysis confirmed that the system with 0\% KLF2 retained bistability (an "Sshaped" curve in Fig. 2e) while the system with 100\% KLF2 was monostable.

\section{The bistability of the system correlates with the sign of the PLS-PAl1 feedback loop}

Positive and negative feedback loops are ubiquitous in biological systems [9], and necessary for many functions $[10,11]$. The TGF- $\beta 1$ activation network is composed of multiple overlapping feedback loops, including two feedback loops between PLS and PAI1. One obvious loop is the negative feedback loop PLS $\rightarrow$ TGF- $\beta 1 \rightarrow$ PAI1 1 PLS, which is frequently cited [12-16]. A less obvious loop is PLS $\dashv$ TSP $1 \rightarrow$ TGF- $\beta 1 \rightarrow$ PAI1 1 PLS, with two inhibitory effects, meaning positive feedback (See Fig. 3. a-b). Interestingly, experiments have already observed two opposite behaviors of PLS towards TGF- $\beta 1$ and PAI1 [17-19], giving indirect evidence for the possibility of both positive and negative feedback loops involving PLS, TGF- $\beta 1$, and PAI1.

To characterize the feedback between PLS and PAI1 in this network, we plotted feedback behavior in both low KLF2 (0\%) and high KLF2 (100\%) models (Fig. 3a). Since PAI1 (plasminogen activator inhibitor-1) is antagonistic towards PLS, the sign of the PLS-PAI1 feedback loop is determined by the response of PAI1 to PLS. We used a stepwise PLS input (black curve) to perturb both the low (\%0) KLF2 model and the high (100\%) KLF2 model and we simulated the response of PAI1. The low KLF2 model showed a decrease in the level of PAI1, which means that the overall PLS-PAI1 feedback is dominated by the PLS $\dashv$ TSP $1 \rightarrow$ TGF- $\beta 1 \rightarrow$ PAI1 $\dashv$ PLS double negative (positive) feedback loop. The high KLF2 model showed an increase in the level of PAI1, which means that the overall PLS-PAI1 feedback is dominated by the $\mathrm{PLS} \rightarrow \mathrm{TGF}-\beta 1 \rightarrow \mathrm{PAI} 1 \dashv \mathrm{PLS}$ negative feedback loop.

It was interesting to observe in simulation that KLF2 not only was able to eliminate one of the steady states and turned the system into monostable, but also able to change the sign of PLS-PAI1 feedback loop. To further characterize the effect of KLF2 on the system and discuss the reason behind it, we plotted the $2 \mathrm{~d}$ bistable region of the system in the $k p 1-k p 2$ phase plane. $k p 1$ is the TSP1 synthesis rate parameter and $k p 2$ is the PAI1 synthesis parameter, both of which affect the bistability of the system. We found the boundaries of the bistable region through equilibrium continuation of $k p 2$ for a series of $k p 1$ values (Fig. 3b). In our model, KLF2 is represented as a combination of fold changes of $k p 1$ and $k p 2$, therefore, KLF2 levels can be represented as a series of points in the $k p 1-k p 2$ phase plane (Fig. 3c). It can be seen on Fig. 3c that KLF2 point is moving out of the bistable region when KLF2 increases from 0 to $100 \%$. We then analyzed the sign of PLS-PAI1 feedback in this $k p 1-k p 2$ phase plane. Interestingly, there is a large overlap between the bistable region of the system and positive feedback region of PLS-PAI1 feedback loop (Fig. $3 d$ ). KLF2 $=0$ point lies in the overlapping area of the bistable region and PLS-PAI1 positive feedback region, while KLF2 $=100 \%$ lies in the overlapping area of the non-bistable region and PLS-PAI1 negative feedback region. This explains why the change of KLF2 level can have two different effects on the system.

The large overlap between bistable region and PLSPAI1 positive feedback region is also an interesting property of the system, since it suggests that in reality, a bistable TGF- $\beta 1$ activation system most likely also has positive PLS-PAI1 feedback. Although negative feedback loop between PLS-PAI1 has been observed repeatedly and is well accepted $[17,18]$, positive feedback would be novel. We tested the sign of the feedback from PLS to PAI1 using an experimental system known to exhibit TGF- $\beta 1$ bistability [5], a cell culture model of liver fibrosis. In this co-culture with primary hepatocytes and HSC-T6 cell lines, we added different levels of PLS and we measured PAI1 mRNA levels using RT-PCR (Fig. $3 \mathrm{~b})$. Increasing PLS was found to cause decreased expression of PAI1 in this bistable system, implying that PLS and PAI1 can indeed exhibit positive feedback.

\section{Discussion}

Using a model of TGF- $\beta 1$ activation, we explored the ability of external factors to switch bistability on and off, and we characterized the correlation between the bistability of the model, and the sign of a feedback loop in the network.

For the first part, we modeled known effects of calcium on the balance between TSP1 and PLS [20-25], and known effects of KLF2 on the gene expression of PAI1 and TSP1 [6]. We then used modeling to show how these effects would propagate through the system. Specifically, the model predicted that calcium would significantly promote TGF- $\beta 1$ activation, shifting the 

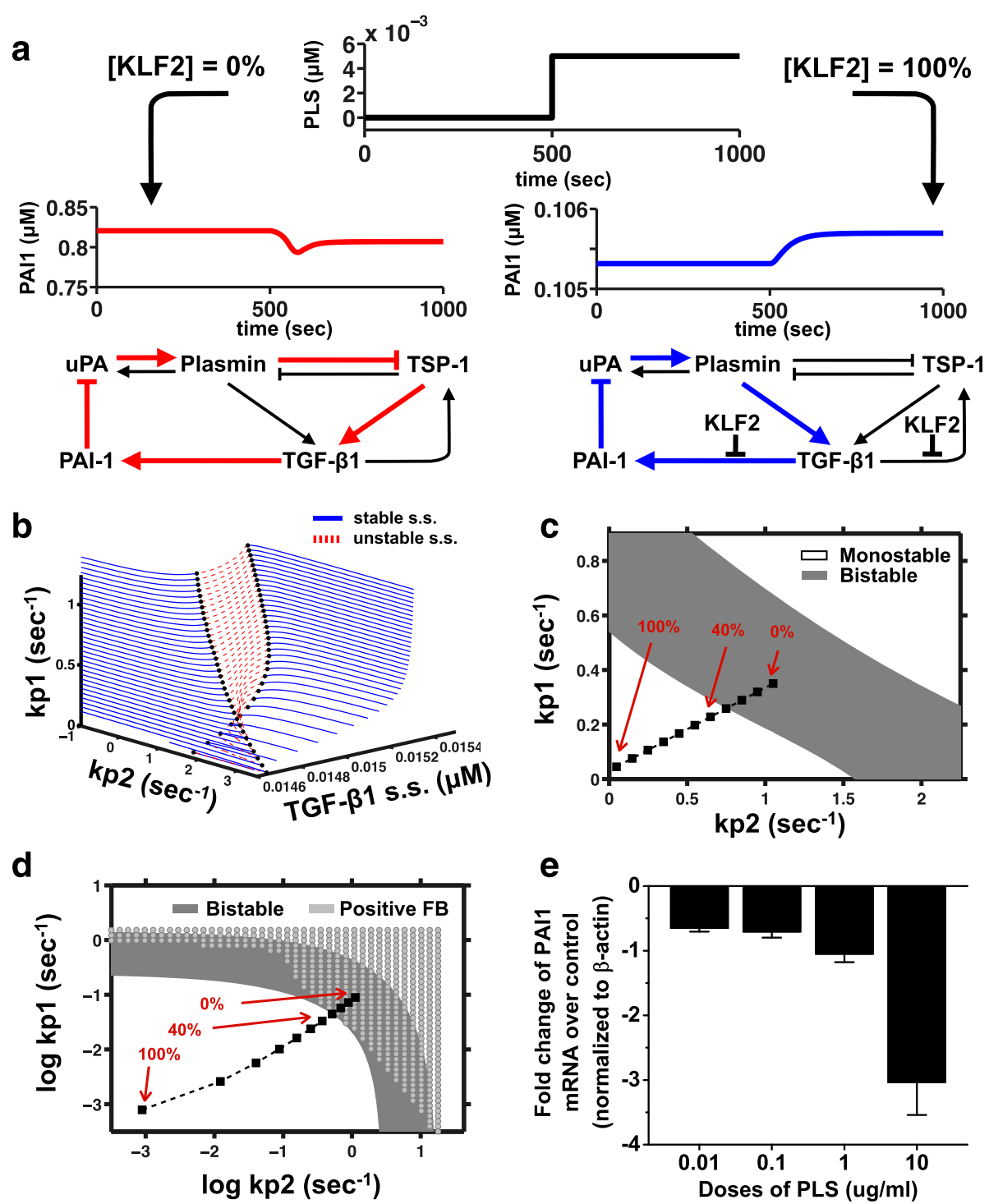

Fig. 3 The bistability of the system correlates with the sign of the PLS-PAl1 feedback loop. a KLF2 affects the sign of PLS-PAl1 feedback loop. We designed an exogenous addition of PLS into the system using a step function for the level of PLS over time (top panel, black curve). Stimulating the TGF- $\beta 1$ activation model with exogenous PLS caused two different effects in silico, depending on the KLF2 status. In the absence of KLF2 (red curve on left), the stimulus caused positive (double-negative) feedback between PAl1 and PLS, which can occur via the red arrows shown. In the presence of KLF2 (blue curve on right), exogenous PLS treatment caused a positive effect on PAl1 and the negative feedback loop (blue arrows) was restored. b Methods to calculate the $2 \mathrm{~d}$ bistable region. We did bifurcation analysis of $\mathrm{kp} 2 \mathrm{for}$ a series of $\mathrm{kp} 1$ value (100 equally spaced values between $\mathrm{kpl}=1.2$ and $\mathrm{kpl}=0$ ) for the TGF- $\beta 1$ activation model. The stable s.s. on the bifurcation curve were plotted as solid blue line, while the unstable s.s. were plotted as dotted red line. The Limit Points (LP), which tell the bistable interval of the bifurcation parameter (kp2) were denoted by black dots on the bifurcation curve. c The series of KLF2 levels cross the boundary of the bistable region in kp 1-kp2 phase plane. The $2 \mathrm{~d}$ bistable region (dark gray) in kp1-kp1 phase plane can be constructed based on the kp2 coordinates of LPs in (b) and their corresponding kp1 values. KLF2 levels are represented as diamond dots. $\mathbf{d}$ Overlap between bistable region and PLS-PAI1 positive feedback region in the kp1-kp2 phase plane. Bistable region is denoted by solid dark gray, while PLS-PAl1 positive feedback region is denoted by dotted light gray. The $x$ and $y$ axis are all in log scale. e Empirically, PLS can down-regulate PAl1 gene expression in a co-culture of hepatocytes and HSC-T6 cells. Hepatic stellate cells (HSC-T6) were cultured with primary rat hepatocytes in a 7:1 ratio overnight, creating a fibrosis-like state with high TGF- $\beta 1$ level. The next day, the medium was changed to non-serum medium with different doses of PLS. Cells were collected $24 \mathrm{~h}$ later

bistable threshold of the system. The calcium-induced increase in TSP1 would lie within the physiological range of TSP1 [26]. Literature search reveals that extracellular calcium may be relatively easy to perturb via biomaterials of bandages, etc. Therefore, the effect of extracellular calcium on TGF- $\beta 1$ might have important therapeutic implications for fibrotic or inflammatory diseases where abnormal TGF- $\beta 1$ contributes to disease. 
For example, fibrotic diseases are driven by high levels of TGF- $\beta 1$ [27], and therapeutic studies in animals have achieved significant access by increasing the PLS pathway [28] or decreasing the TSP1 pathway [29]. In our model, if we take the ssP state to be healthy and the ssT state to be fibrotic, then a fibrotic system with high calcium could transition toward health through an increase of PLS (Fig. 2c, arrow 1), through a decrease of TSP1 (Fig. 2c, arrow 2), or a combination of both (Fig. 2c, arrow 3).

In contrast, KLF2 was simulated to increase PLS activity and decrease the levels of TGF- $\beta 1$, by suppressing PAI1 and TSP1 expression. This is consistent with previous work with statin drugs on liver fibrosis [30], where KLF2 upregulation was observed after treatment with simvastatin. Our model predicts that one of the ways KLF2 may contribute to improvement of liver fibrosis may be by decreasing the activation of TGF- $\beta 1$ through reduction of the TSP1 and PAI feedback effects.

While modeling KLF2 effects, we noticed that loss of bistability also caused a change in the sign of the PLS PAI1 feedback loop. Without KLF2, the PLS-PAI1 feedback loop was positive (double negative), but with KLF2 (100\% KLF2) and with the destruction of bistability, the PLS-PAI1 feedback loop was negative. Additional bifurcation analysis revealed that high KLF2 is a special case of the general observation, that the bistability of the system is correlated with the sign of the PLS-PAI1 feedback loop.

We demonstrated the positive feedback behavior between PLS and PAI1 in the bistable TGF- $\beta 1$ system, using an in vitro experiment. Previous studies have already revealed two opposite behaviors of PLS towards TGF- $\beta 1$ and PAI1 [17-19], providing indirect evidence for the possibility of both positive and negative feedback loops. Some aspects of the feedback loop are relatively unambiguous. For example, PAI1 is a specific and potent inhibitor of plasmin activation. PAI1 production follows TGF- $\beta 1$ signaling so closely that, in practice, PAI1 levels are commonly measured as a readout of TGF- $\beta 1$ activation [31]. The behavior of the feedback loop thus boils down to the behavior of the PLS - TGF- $\beta 1$ relationship. In isolation, PLS clearly is able to activate TGF- $\beta 1$. The same effect has frequently been observed in more physiological contexts, and there is considerable published evidence that PLS and/or plasminogen activators can cause an increase in TGF- $\beta 1$ and/or PAI1 levels [12-16]. This positive effect of PLS on TGF- $\beta 1$ or PAI1 serves as evidence that the loop between PLS and PAI1 can have negative feedback. Although the activating ability of PLS toward TGF- $\beta 1$ is well known and accepted, some studies also suggest the opposite effect. For example, PLS caused TGF- $\beta 2$ levels to decline in breast cysts [32]. Furthermore, one unconventional finding by
Seo et al., showed a positive feedback effect between PAI1 and TGF- $\beta 1$ [19], suggesting that PLS can cause a decrease in TGF- $\beta 1$. In sum, we found that there is some support in the published literature for our prediction that the relationship between PLS and PAI can show either negative feedback or positive feedback, depending on context.

Another important consideration in interpreting this model is the redundancy of proteases and matrix factors that play roles similar to PLS or TSP1. Actually, PLS is only one of many proteases (including elastase, MMP-2, MMP-9, ADAMTS1 and others) that can both activate TGF- $\beta 1$ and cleave TSP1 [33-36]. Meanwhile TSP1 can inhibit many of these proteases [20,37-39]. Extracellular proteases often function interdependently by activating each other (e.g. PLS activates several MMPs, which activate other MMPs [40, 41]), and some proteases may have partially redundant effects. Likewise TSP1 may represent a larger class of matrix proteins and mechanical factors with redundant roles in this model. Fibrillin and LTBP1 can promote TGF- $\beta 1$ activation [42-45], as can factors that create mechanical tension in the matrix $[46,47]$. Fibrillin and LTBP1 can be cleaved by PLS $[14,48]$, and even mechanical tension would be antagonized by PLS cleavage. In other words, PLS and TSP1 are archetypes of two larger classes of effects, a protease category and a matrix category, that may be capable of antagonizing the effect of each other, even as they contribute individually to TGF- $\beta 1$ activation. The redundancy of the protease-versus-matrix competition suggests that this antagonism may be an organizing principle of TGF- $\beta 1$ regulation, with evolutionary importance to the organism. On the other hand, this redundancy also creates many complexities that could perturb the phenomena we simulated. For example, the effects we attribute to PLS itself may actually result from the indirect effects of PLS-activated proteases. Thus, we speculate that the insights we drew from this model point to important properties of general TGF- $\beta 1$ activation regulatory networks in different contexts.

\section{Conclusions}

Using TGF- $\beta$ as a case study, we demonstrated that external factors could influence the bistability of the model, and that these influences can be modeled implicitly using the reaction parameters of the model. Furthermore, we showed that system-level properties like the sign of feedback loops can correlate with the bistability of a complex model. This provides a novel characterization of the transition between bistable and monostable regimes, and provides a non-obvious explanation for seemingly contradictory experimental findings 
about the contribution of the PAI1 - PLS feedback loop toward TGF- $\beta$ activation.

\section{Methods}

TGF- $\beta 1$ bistable activation model

We used the model built by Venkatraman et al. as the base model of TGF- $\beta 1$ regulation. We increased the " $k_{\text {others }}$ " parameter relative to the published model, to allow for higher basal activation of TGF- $\beta 1$ by other activators such as integrins $[42,49,50]$. Simulations were performed using kroneckerbio toolbox [51] and the ode15s solver in MATLAB (Mathworks, Natick, MA).

\section{Calcium model}

Calcium can affect the structure of TSP1 [22-25], the enzymatic activity of PLS cleaving TSP1 [20], and the ability of TSP1 to inhibit serine protease activity [21]. These effects were represented by the rate constants k3, k_3 and $\mathrm{k} 4$ in the TGF- $\beta 1$ activation model. We used the original parameter settings as the low calcium settings. To reflect high calcium conditions, we increased $\mathrm{k} 3$ by 10 fold, decreased k_3 by 0.1 fold, and k4 by 0.0001 fold, in order to reflect a high level of calcium in the environment. Details of the model can be found in Table S1 (Additional file 2) and Table S2 (Additional file 3).

\section{KLF2 model}

It has been shown that KLF2 can decrease TSP1 expression by 7.8 fold and PAI1 expression by 7.4 fold $[6,7]$. We simulated the TGF- $\beta 1$ activation model with no change $(0 \%$ of the KLF2 effect, original parameter settings), with $100 \%$ of the KLF2 effect (7.4 fold decrease of PAI1 synthesis parameter $k p 2$ and 7.8 fold decrease of TSP1 synthesis parameter $k p 1$ ), as well as a series of intermediate models with $10 \%, 20 \%, \ldots 90 \%$ of the KLF2 effect, causing intermediate levels of decrease in the PAI1 and TSP1 synthesis rates.

\section{Bifurcation analysis}

Bifurcation analysis was performed using MATCONT (https://sourceforge.net/projects/matcont/). Equilibrium continuation function was called to generate the bifurcation curves in Fig. 2e and Fig. 3b.

\section{Experimental methods}

Isolation of primary hepatocytes was performed on male Wistar rats (250-300 g), via a two-step collagenase perfusion method as described previously [52]. A co-culture model of primary rat hepatocytes and hepatic stellate cell line T6 (HSC-T6) was established as described in [5]. Briefly, primary rat hepatocytes were first seeded at a density of $2 \times 10^{5}$ cells on $35 \mathrm{~mm}$ collagen-coated dishes (IWAKI) using Williams's E media with 10\% FBS. After $4 \mathrm{~h}$, hepatic stellate cell line T6 (HSC-T6) was seeded at a density of $1.4 \times 10^{6}$ cells. The cells were cultured overnight in $35{ }^{\circ} \mathrm{C}$, and $5 \% \mathrm{CO}_{2}$ in William's $\mathrm{E}$ media with $2 \% \mathrm{FBS}$ to facilitate HSC activation. The next day media was changed to Williams's E without serum, along with different doses of PLS. After $24 \mathrm{~h}$, the cells were collected.

RT-PCR was performed as described in [53]. Briefly, mRNA was isolated from the cells using RNeasy mini kit (Qiagen), and its concentration was quantified using a Nanodrop 2000 UV-Vis Spectrophotometer. One microgram of mRNA from each sample was converted to cDNA (Invitrogen, Superscript Reverse Transcriptase III) and real-time PCR reaction (Roche, Sybr Green Master mix) was carried out for plasminogen activator inhibitor-1 (PAI1) and $\beta$-actin, with in-house primers shown in Table S3 (Additional file 4). The gene expression values were determined by the Del-Del $C_{T}$ relative quantitation method; the target $C_{T}$ values were normalized to the endogeneous reference $\beta$-actin, and the normalized mRNA was expressed as a fold-change relative to the untreated control.

\section{Additional files}

Additional file 1: Supplementary Notes. (PDF 107 kb)

Additional file 2: Table S1. List of equations and parameters used for model construction. (PDF $280 \mathrm{~kb}$ )

Additional file 3: Table S2. Parameters settings for different models. (PDF $47 \mathrm{~kb}$ )

Additional file 4: Table S3. List of primer sequences for genes probed on quantitative real time PCR. (PDF $26 \mathrm{~kb}$ )

\section{Abbreviations}

PAI1: Plasminogen activator inhibitor-1; PLG: Plasminogen; PLS: Plasmin; ssP: PLS predominant steady state; SsT: TSP1 predominant steady state; TGF$\beta 1$ : Transforming growth factor $\beta 1$; TSP1: Thrombospondin-1

\section{Acknowledgements}

We thank members of the Singapore-MIT Alliance for helpful suggestions. $\mathrm{HL}$ and BCN have since moved to the Genome Institute of Singapore, 60 Biopolis Street, 138672 Singapore, and LV to the Department of Immunology, Genetics and Pathology, Dag Hammarskjölds väg 20, Rudbeck Laboratory, 75185 Uppsala, Sweden.

\section{Funding}

Publication costs were paid by the Singapore Ministry of Health's National Medical Research Council (NMRC) under its Open Fund Individual Research Grant scheme (OFIRG15nov062) to LTK. This work was also supported by a Singapore-MIT Alliance Computational and Systems Biology Flagship Project grant; by Singapore-MIT Alliance Computational and Systems Biology Programme grants to LTK, JKW, and HY; by funding from the Mechanobiology Institute, the Institute of Bioengineering and Nanotechnology, and grants from Jansson-Cilag Singapore (R-185-000-182-592) to HY.

\section{Availability of data and materials}

All data generated or analysed during this study are included in this published article and its supplementary information files.

\section{About this supplement}

This article has been published as part of BMC Systems Biology Volume 11 Supplement 7, 2017: 16th International Conference on Bioinformatics (InCoB 2017): Systems Biology. The full contents of the supplement are available 
online at https://bmcsystbiol.biomedcentral.com/articles/supplements/ volume-11-supplement-6.

\section{Authors' contributions}

$\mathrm{HL}$ constructed the models, ran simulations and generated graphs. LTK and HY conceived of the study and identified literature sources. LV performed the co-culture and cell treatments. BCN performed the RT-PCR. HL and LTK wrote the paper. All authors provided critical evaluation of the manuscript.

\section{Ethics approval and consent to participate}

The rat cell isolation procedure was approved by the IACUC of the National University of Singapore.

\section{Consent for publication}

Not applicable.

\section{Competing interests}

The authors declare that they have no competing interests.

\section{Publisher's Note}

Springer Nature remains neutral with regard to jurisdictional claims in published maps and institutional affiliations.

\section{Author details \\ ${ }^{1}$ Computational and Systems Biology Program, Singapore-MIT Alliance, Singapore 117576, Singapore. ${ }^{2}$ Mechanobiology Institute, National University of Singapore, Singapore 117411, Singapore. ${ }^{3}$ NUS Graduate School for Integrative Sciences and Engineering, National University of Singapore, Singapore 117456, Singapore. ${ }^{4}$ Department of Physiology, National University of Singapore, Singapore 117597, Singapore. ${ }^{5}$ Department of Electrical Engineering and Computer Science, Massachusetts Institute of Technology, Cambridge, MA 02139, USA. 'BioSystems and Micromechanics IRG, Singapore-MIT Alliance for Research and Technology, Singapore 138602, Singapore. ${ }^{7}$ Institute of Bioengineering and Nanotechnology, A*STAR, Singapore 138669, Singapore. ${ }^{8}$ Department of Biological Engineering, Massachusetts Institute of Technology, Cambridge, MA 02139, USA. ${ }^{9} \mathrm{Center}$ for Computational Biology, Duke-NUS Medical School, Singapore 169857, Singapore. ${ }^{10}$ Cancer and Stem Cell Biology, Duke-NUS Medical School, Singapore 169857, Singapore.}

\section{Published: 21 December 2017}

\section{References}

1. Albeck JG, Burke JM, Spencer SL, Lauffenburger DA, Sorger PK. Modeling a snap-action, variable-delay switch controlling extrinsic cell death. PLoS Biol. 2008:6:2831-52.

2. Shi Y, Mellier G, Huang S, White J, Pervaiz S, Tucker-Kellogg L. Computational modelling of LY303511 and TRAlL-induced apoptosis suggests dynamic regulation of cFLIP. Bioinformatics. 2013:29:347-54.

3. Chatterjee A, Kaznessis YN, W-S H. Tweaking biological switches through a better understanding of bistability behavior. Curr Opin Biotechnol. 2008;19:475-81.

4. Sobie EA. Bistability in Biochemical Signaling Models. Sci Signal. 2011;4:tr10

5. Venkatraman L, Chia S-M, Narmada BC, White JK, Bhowmick SS, Forbes Dewey C Jr, So PT, Tucker-Kellogg L, Yu H. Plasmin triggers a switch-like decrease in Thrombospondin-dependent activation of TGF- $\beta 1$. Biophys J. 2012;103:1060-8.

6. Dekker RJ. KLF2 provokes a gene expression pattern that establishes functional quiescent differentiation of the endothelium. Blood. 2006;107: 4354-63.

7. Boon RA, Fledderus JO, Volger OL, van Wanrooij EJA, Pardali E, Weesie F, Kuiper J, Pannekoek H, Dijke Ten P, AJG H. KLF2 suppresses TGF-beta signaling in endothelium through induction of Smad7 and inhibition of AP1. Arterioscler Thromb Vasc Biol. 2007;27:532-9.

8. Strogatz SH. Nonlinear dynamics and chaos. Westview Press. 2008;352

9. Neves S, lyengar R. Modeling of signaling networks - Neves - 2002 BioEssays - Wiley online library. BioEssays. 2002;

10. Ratushny AV, Saleem RA, Sitko K, Ramsey SA, Aitchison JD. Asymmetric positive feedback loops reliably control biological responses. Mol Syst Biol. 2012;8:577.
11. Tsai TY-C, Choi YS, Ma W, Pomerening JR, Tang C, Ferrell JE. Robust, tunable biological oscillations from interlinked positive and negative feedback loops. Science. 2008;321:126-9.

12. Grainger DJ, Wakefield L, Bethell HW, Farndale RW, Metcalfe JC. Release and activation of platelet latent TGF-beta in blood clots during dissolution with plasmin. Nat Med. 1995;1:932-7.

13. Khalil N, Corne S, Whitman C, Yacyshyn H. Plasmin regulates the activation of cell-associated latent TGF-beta 1 secreted by rat alveolar macrophages after in vivo bleomycin injury. Am J Respir Cell Mol Biol. 1996;15:252-9.

14. Yee JA, Yan L, Dominguez JC, Allan EH, Martin TJ. Plasminogen-dependent activation of latent transforming growth factor beta (TGF beta) by growing cultures of osteoblast-like cells. J Cell Physiol. 1993;157:528-34.

15. Godár S, Horejsi V, Weidle UH, Binder BR, Hansmann C, Stockinger H. M6P/ IGFII-receptor complexes urokinase receptor and plasminogen for activation of transforming growth factor-beta1. Eur J Immunol. 1999:29:1004-13.

16. Binder BR, Christ G, Gruber F, Grubic N, Hufnagl P, Krebs M, Mihaly J, Prager GW. Plasminogen activator inhibitor 1: physiological and pathophysiological roles. News Physiol Sci. 2002;17:56-61.

17. Thornton AJ, Bruzdzinski CJ, Raper SE, Gelehrter TD. Plasminogen activator inhibitor-1 is an immediate early response gene in regenerating rat liver. Cancer Res. 1994:54:1337-43.

18. Flaumenhaft R, Abe M, Mignatti P, Rifkin DB. Basic fibroblast growth factorinduced activation of latent transforming growth factor beta in endothelial cells: regulation of plasminogen activator activity. J Cell Biol. 1992;118:901-9.

19. Seo JY, Park J, Yu MR, Kim YS, Ha H, Lee HB. Positive feedback loop between plasminogen activator Inhibitor-1 and transforming growth factorBeta1 during renal fibrosis in diabetes. Am J Nephrol. 2009;30:481-90.

20. Hogg PJ, Stenflo J, Mosher DF. Thrombospondin is a slow tight-binding inhibitor of plasmin. Biochemistry. 1992;31:265-9.

21. Gotis-Graham I, Hogg PJ, McNeil HP. Significant correlation between thrombospondin 1 and serine proteinase expression in rheumatoid synovium. Arthritis Rheum. 1997:40:1780-7.

22. Calzada MJ, Kuznetsova SA, Sipes JM, Rodrigues RG, Cashel JA, Annis DS, Mosher DF, Roberts DD. Calcium indirectly regulates immunochemical reactivity and functional activities of the $\mathrm{N}$-domain of thrombospondin-1. Matrix Biol. 2008;27:339-51.

23. Dixit VM, Galvin NJ, O'Rourke KM, Frazier WA. Monoclonal antibodies that recognize calcium-dependent structures of human thrombospondin. Characterization and mapping of their epitopes. J Biol Chem. 1986;261: 1962-8.

24. Misenheimer TM, Mosher DF. Calcium ion binding to thrombospondin 1. J Biol Chem. 1995;270:1729-33.

25. Slane JM, Mosher DF, Lai CS. Conformational change in thrombospondin induced by removal of bound Ca2+. A spin label approach. FEBS Lett. 1988; 229:363-6.

26. Bergseth $G$, Lappegård KT, Videm V, Mollnes TE. A novel enzyme immunoassay for plasma thrombospondin. Comparison with betathromboglobulin as platelet activation marker in vitro and in vivo. Thromb Res. 2000;99:41-50.

27. Branton MH, Kopp JB. ScienceDirect.com - microbes and infection - TGF- $\beta$ and fibrosis. Microbes Infect. 1999;1:1349-65.

28. Wang $\mathrm{H}$, Zhang $\mathrm{Y}$, Heuckeroth RO. PAl-1 deficiency reduces liver fibrosis after bile duct ligation in mice through activation of tPA. FEBS Lett. 2007; 581:3098-104

29. Hugo C. The thrombospondin 1-TGF-beta axis in fibrotic renal disease. Nephrol Dial Transplant. 2003;18:1241-5.

30. Marrone G, Russo L, Rosado E, Hide D, García-Cardeña G, García-Pagán JC, Bosch J, Gracia-Sancho J. The transcription factor KLF2 mediates hepatic endothelial protection and paracrine endothelial-stellate cell deactivation induced by statins. J Hepatol. 2012;

31. Mazzieri R, Munger JS, Rifkin DB. Measurement of active TGF-beta generated by cultured cells. Methods Mol Biol. 2000;142:13-27.

32. Erbas $\mathrm{H}$, Erten $\mathrm{O}$, Irfanoglu ME. Breast cyst fluid plasmin activity and its effect on TGF-beta2 activation. Cancer Investig. 2008;26:22-7.

33. Bourd-Boittin K, Bonnier D, Leyme A, Mari B, Tuffery P, Samson M, Ezan F, Baffet $G$, Theret N. Protease profiling of liver fibrosis reveals the ADAM metallopeptidase with thrombospondin type 1 motif, 1 as a central activator of transforming growth factor beta. Hepatology. 2011:54:2173-84.

34. Sato Y, Rifkin DB. Inhibition of endothelial cell movement by pericytes and smooth muscle cells: activation of a latent transforming growth factor-beta 1-like molecule by plasmin during co-culture. J Cell Biol. 1989;109:309-15. 
35. Yu Q, Stamenkovic I. Cell surface-localized matrix metalloproteinase-9 proteolytically activates TGF-beta and promotes tumor invasion and angiogenesis. Genes Dev. 2000;14:163-76.

36. Prudova A, Auf Dem Keller U, Butler GS. Overall CM: multiplex N-terminome analysis of MMP-2 and MMP-9 substrate degradomes by iTRAQ-TAlLS quantitative proteomics. Mol Cell Proteomics. 2010;9:894-911.

37. Lawler PR, Lawler J. Molecular basis for the regulation of angiogenesis by thrombospondin-1 and -2. Cold Spring Harb Perspect Med. 2012;2:a006627.

38. Hogg PJ, Owensby DA, Chesterman CN. Thrombospondin 1 is a tightbinding competitive inhibitor of neutrophil cathepsin G. Determination of the kinetic mechanism of inhibition and localization of cathepsin $\mathrm{G}$ binding to the thrombospondin 1 type 3 repeats. J Biol Chem. 1993;268:21811-8.

39. Bein K, Simons M. Thrombospondin type 1 repeats interact with matrix metalloproteinase 2. Regulation of metalloproteinase activity. J Biol Chem. 2000;275:32167-73.

40. Davis GE, Pintar Allen KA, Salazar R, Maxwell SA. Matrix metalloproteinase-1 and -9 activation by plasmin regulates a novel endothelial cell-mediated mechanism of collagen gel contraction and capillary tube regression in three-dimensional collagen matrices. J Cell Sci. 2001;114:917-30.

41. Curry TE, Osteen KG. The matrix metalloproteinase system: changes, regulation, and impact throughout the ovarian and uterine reproductive cycle. Endocr Rev. 2003;24:428-65.

42. Annes JP, Chen Y, Munger JS, Rifkin DB. Integrin alphaVbeta6-mediated activation of latent TGF-beta requires the latent TGF-beta binding protein-1. J Cell Biol. 2004;165:723-34.

43. Kaartinen V Warburton D. Fibrillin controls TGF-beta activation. Nat Genet. 2003;33:331-2.

44. Tritschler I, Gramatzki D, Capper D, Mittelbronn M, Meyermann R, Saharinen J, Wick W, Keski-Oja J, Weller M. Modulation of TGF-beta activity by latent TGF-beta-binding protein 1 in human malignant glioma cells. Int J Cancer. 2009;125:530-40.

45. Chaudhry SS, Cain SA, Morgan A, Dallas SL, Shuttleworth CA, Kielty CM. Fibrillin-1 regulates the bioavailability of TGFbeta1. J Cell Biol. 2007;176:355-67.

46. Buscemi L, Ramonet D, Klingberg F, Formey A, Smith-Clerc J, Meister J-J, Hinz B. The single-molecule mechanics of the latent TGF- $\beta 1$ complex. Curr Biol. 2011;21:2046-54.

47. Ahamed J, Janczak CA, Wittkowski KM, Coller BS. PLOS ONE: in vitro and in vivo evidence that Thrombospondin-1 (TSP-1) contributes to stirring- and shear-dependent activation of platelet-derived TGF-B1. PLoS One. 2009;4: e6608.

48. Kirschner R, Hubmacher D, lyengar G, Kaur J, Fagotto-Kaufmann C, Brömme D, Bartels R, Reinhardt DP. Classical and neonatal Marfan syndrome mutations in fibrillin-1 cause differential protease susceptibilities and protein function. J Biol Chem. 2011;286:32810-23.

49. Annes JP, Munger JS, Rifkin DB. Making sense of latent TGFbeta activation. J Cell Sci. 2003:116:217-24

50. Fontana L, Chen Y, Prijatelj P, Sakai T, Fässler R, Sakai LY, Rifkin DB. Fibronectin is required for integrin alphavbeta6-mediated activation of latent TGF-beta complexes containing LTBP-1. FASEB J. 2005;19:1798-808.

51. Toettcher JE, Apgar JF, Castillo AR, Tidor B, White J. Simulation and verification of electronic and biological systems. Dordrecht: Springer Netherlands; 2011. p. 115-36.

52. Seglen PO. Preparation of isolated rat liver cells. Methods Cell Biol. 1976;13: 29-83.

53. Narmada BC, Chia S-M, Tucker-Kellogg L, Yu H. HGF regulates the activation of TGF- $\beta 1$ in rat hepatocytes and hepatic stellate cells. J Cell Physiol. 2012; 228:393-401.

\section{Submit your next manuscript to BioMed Central and we will help you at every step:}

- We accept pre-submission inquiries

- Our selector tool helps you to find the most relevant journal

- We provide round the clock customer support

- Convenient online submission

- Thorough peer review

- Inclusion in PubMed and all major indexing services

- Maximum visibility for your research

Submit your manuscript at www.biomedcentral.com/submit

) Biomed Central 